\title{
Blunt needle revision with viscoelastic materials via the anterior chamber for early failed filtering blebs after trabeculectomy
}

This article was published in the following Dove Press journal:

Clinical Ophthalmology

14 June 2012

Number of times this article has been viewed

\section{Nozomi Kinoshita \\ Ayumi Ota \\ Fumihiko Toyoda \\ Hiroko Yamagami \\ Akihiro Kakehashi}

Department of Ophthalmology,

Saitama Medical Center, Jichi Medical

University, Saitama, Japan
Correspondence: Akihiro Kakehashi Department of Ophthalmology, Saitama Medical Center, Jichi Medical University, I-847 Amanuma-cho,

Omiya-ku, Saitama, 330-8503, Japan

Tel +8I 486472 III

Fax +81486485188

Email kakeaki@omiya.jichi.ac.jp
Purpose: To report a new technique of blunt needle revision with viscoelastic materials via the anterior chamber for the treatment of early failed filtering blebs and elevated intraocular pressure after trabeculectomy, in which digital ocular massage and laser suture lysis have been ineffective.

Methods: A 27-gauge blunt needle attached to a syringe containing viscoelastic material was inserted into the anterior chamber from the inferior paracentesis. The needle tip was inserted into the subscleral flap space from the filtering fistula at the anterior chamber side, and the scleral flap was lifted bluntly. The needle tip was then inserted into the subconjunctival space where the viscoelastic agent was injected and the adhesion between the sclera and conjunctiva was separated bluntly. Blunt needle revision via the anterior chamber was performed 14 times in six eyes of six patients at Saitama Medical Center, Jichi Medical University from January 2007 to May 2009. All procedures were performed within 1 month after trabeculectomy.

Results: The intraocular pressure remained $21 \mathrm{mmHg}$ or lower for more than 6 months in three of six eyes. Slight bleeding from the iris occurred in one of the 14 procedures, and hypotony (intraocular pressure below $5 \mathrm{mmHg}$ ) occurred in one of the 14 procedures. No serious complications developed.

Conclusion: Blunt needle revision via the anterior chamber for early failed filtering blebs is a new, simple, and safe procedure.

Keywords: glaucoma, trabeculectomy, filtering bleb, needle revision, blunt needle

\section{Introduction}

When failed filtration causes elevated intraocular pressure (IOP) after trabeculectomy, digital ocular massage ${ }^{1,2}$ and laser suture $\operatorname{lysis}^{2,3}$ may be performed. If ineffective, the next intervention is a minimally invasive reparative surgery of the filtering bleb by transconjunctival needle revision. ${ }^{1,2,4}$ Finally, if filtration is still inadequate, an invasive reparative surgery of the filtering bleb or another filtering procedure at a new site may be required. ${ }^{1,5}$

Transconjunctival needle revision procedures for failed filtering blebs have been performed widely. ${ }^{5-7}$ Reported complications that may occur during and after the procedure include: diminished intraoperative visibility, postoperative recurrence of adhesions secondary to subconjunctival hemorrhage, ${ }^{5}$ and postoperative leakage from the filtering bleb as a result of conjunctival perforation with a sharp needle and separation and incision of the adhesion site..$^{5-7}$ There is a risk of complications such as scleral perforation and iris and crystalline lens damage, as the procedure involves separation of adhesions under the scleral flap with minimal visualization. 
We developed and performed a needle revision procedure via the anterior chamber using a blunt needle with injection of viscoelastic materials to treat patients with early failed filtering blebs and elevated IOP after trabeculectomy despite digital ocular massage and laser suture lysis. The aims of this study were to evaluate the effectiveness and safety profile of this method.

\section{Materials and methods}

This is an interventional case series of consecutive patients with early failed filtering blebs treated with blunt needle revision, with a follow up of longer than 6 months from January 2007 to May 2009. We defined early failed filtering bleb as flat bleb with elevated IOP despite digital ocular massage and laser suture lysis within 1 month. We defined success of needle revision as postoperative IOP of $21 \mathrm{mmHg}$ or lower for more than 6 months with or without medication. Initial trabeculectomy was performed with application of $0.04 \%$ mitomycin $\mathrm{C}(\mathrm{MMC})$ for 3 minutes under the conjunctiva around the scleral flap. All patients provided written informed consent and one surgeon (AK) performed all surgeries. The institutional review board of Saitama Medical Center, Jichi Medical University, approved the present study in accordance with the tenets of the Declaration of Helsinki.

All blunt needle revision procedures were performed within 1 month following filtration surgery. The schema of the blunt needle revision via the anterior chamber for the failed filtering bleb is shown in Figure 1. A paracentesis was created with perforation of the anterior chamber from the inferior corneal limbus. A 27-gauge blunt needle attached to a syringe containing viscoelastic materials (Opegan Hi; Santen Pharmaceutical Co, Ltd, Osaka, Japan) was inserted into the anterior chamber through the inferior paracentesis. The angle was widened by

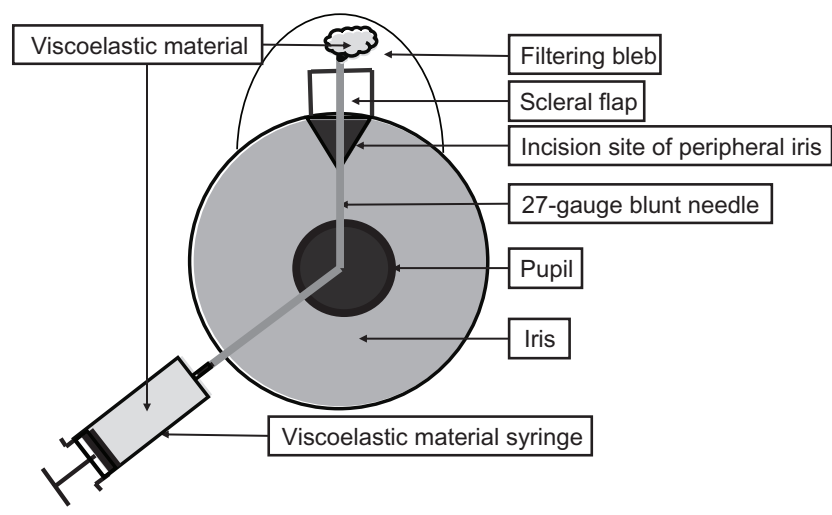

Figure I The schema of the blunt needle revision via the anterior chamber to treat failed filtering blebs.

Notes: A 27-gauge blunt needle tip attached to a syringe containing viscoelastic materials is inserted via the anterior chamber into the subscleral flap space. The adhesion of the scleral flap is separated bluntly. Finally, the adhesion between the sclera and conjunctiva is separated bluntly with viscoelastic materials. injecting a small amount of viscoelastic material, before the blunt needle tip was inserted into the subscleral flap space from the filtering fistula at the site of the peripheral iridectomy created during the previous trabeculectomy. The scleral flap was lifted bluntly. The needle tip was then inserted into the subconjunctival space where the viscoelastic materials were injected and the adhesion between the sclera and conjunctiva separated bluntly. The eyeball was pressed slightly while injecting the intraocular perfusate into the anterior chamber to confirm adequate flow from the anterior chamber into the filtering bleb at the completion of the surgery (Figure 2). Antimetabolites such as MMC or 5-fluorouracil (5-FU) were not used during blunt needle revision.

\section{Results}

There were 14 needle revision procedures performed in six eyes of six patients included in the present study. Initial trabeculectomy was performed on 40 eyes of 33 patients. Blunt needle revision for a failed filtering bleb was performed on three eyes of three patients after the first trabeculectomy and three eyes of three patients after the second filtration surgery. Two eyes of two patients required several needle revision procedures. Seven needle revisions were performed in case 1 and three needle revisions in case 2 . In both cases, the primary disease was uveitis (Table 1) and the IOP was well maintained for a while; however, the filtering bleb soon adhered again with a subsequent increase in the IOP.
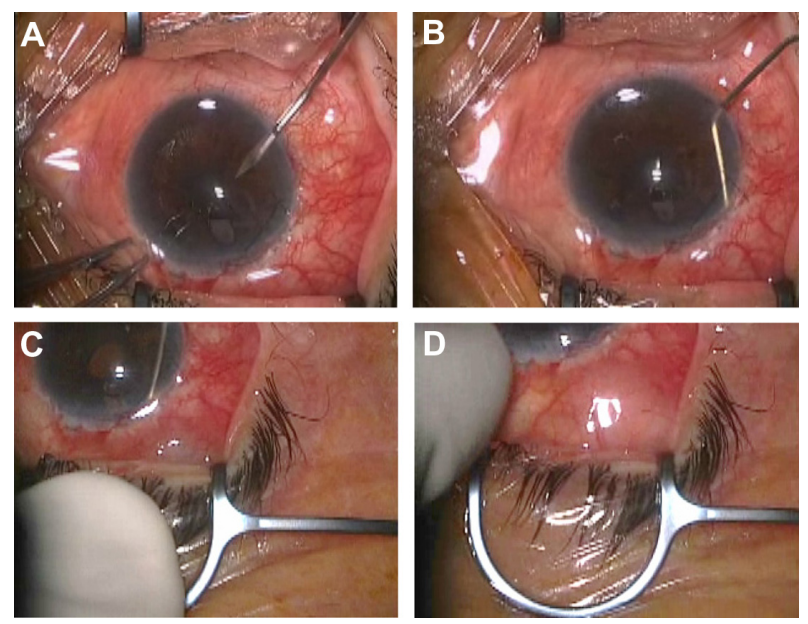

Figure 2 The procedures of the blunt needle revision technique via the anterior chamber to treat failed filtering blebs. A paracentesis is created by perforating the anterior chamber from the inferior corneal limbus (A). A 27-gauge blunt needle attached to a syringe containing viscoelastic materials is inserted into the anterior chamber. The blunt needle tip is then inserted into the subscleral flap space from the filtering fistula, and the scleral flap is lifted bluntly (B). The needle tip is then inserted into the subconjunctival space where the viscoelastic material is injected, and the adhesion between the sclera and conjunctiva separated bluntly (C). The eyeball is pressed slightly to confirm adequate flow from the anterior chamber into the filtering bleb (D). 
Table I Primary diseases in patients who underwent blunt needle revision via the anterior chamber for failed filtering blebs

\begin{tabular}{lllll}
\hline Case & Sex & Age (years) & Primary disease & Eye \\
\hline I & Male & 49 & Uveitis (traumatic) & Right \\
2 & Female & 63 & Uveitis (Harada disease) & Right \\
3 & Male & 39 & Steroid (atopic dermatitis) & Right \\
4 & Male & 61 & Neovascular (diabetes) & Right \\
5 & Female & 52 & Neovascular (diabetes) & Right \\
6 & Male & 60 & Primary open-angle glaucoma & Left \\
\hline
\end{tabular}

The demographics and clinical findings of patients in this study are summarized in Table 1. The male-to-female ratio was $4: 2$ and the patient age (mean \pm standard deviation [SD]) was $54.0 \pm 9.1$ (range, 39-63; median, 56). The primary diseases of eyes that required needle revision were uveitis in two patients, diabetes-associated neovascularization in two patients, steroid-associated glaucoma as a result of treatment for atopic dermatitis in one patient, and primary open-angle glaucoma in one patient. The preoperative IOP (mean $\pm \mathrm{SD}$ ) was $30.8 \pm 10.2 \mathrm{mmHg}$ (range, 19-52; median, 27). The time (mean $\pm \mathrm{SD}$ ) from the filtration surgery including invasive repair to the first needle revision was $10.6 \pm 6.6$ days (range, $2-19$; median, 13). The surgical duration (mean $\pm \mathrm{SD}$ ) of needle revision was $8.3 \pm 4.6$ minutes (range, $2-18$; median, 6.5 ).

Postoperatively, three of six cases (cases 4,5 , and 6 ) had an IOP of $21 \mathrm{mmHg}$ or lower for more than 6 months. Case 4 did not use medications, case 5 used three medications, and case 6 used four medications. Three of six cases (cases 1, 2, and 3) ultimately required an additional surgery. Five refiltration procedures were performed in case 1; cyclocryopexy was performed in one surgery. Three refiltration procedures each were performed in cases 2 and 3.

Intraoperative complications with high incidence rates that develop during transconjunctival needle revision, ${ }^{5,6}$ such as subconjunctival hemorrhage, blood inflow into the anterior chamber, and aqueous leakage from the needling site, did not occur in the current study. The following postoperative complications occurred in one procedure for each of the total 14 needle revision procedures performed: slight bleeding from the iris and hypotony (IOP below $5 \mathrm{mmHg}$ ), which continued for 1 week postoperatively. Severe complications including filtering bleb leak, shallowing of the anterior chamber, choroidal detachment, or postoperative infections did not develop in any of the procedures.

\section{Discussion}

Two studies in the literature have reported needle revision via the anterior chamber for failed filtering blebs. Yokoyama et al reported a series of needle revision surgeries performed on three eyes of two patients. The investigators inserted a sharp 24-gauge needle into the subscleral flap from the filtering fistula at the anterior chamber side. The scleral flap was then lifted and intraocular perfusate was injected to separate the sclera and conjunctiva. ${ }^{8}$ Pasternack et al reported a case series of needle revision procedures performed on 52 eyes of 52 patients. After a subconjunctival injection of 5-FU and transconjunctival needle revision, a cyclodialysis spatula was passed across the anterior chamber through the inferior paracentesis and inserted into the subscleral flap space and subconjunctival space from the filtering fistula at the anterior chamber side. The connection between the anterior chamber and the subconjunctival space was confirmed and enlarged. ${ }^{9}$

The procedure in the current study differed from that described in these two series in two ways. Firstly, the current needle revision procedure was performed only during the early postoperative stage (within 1 month) after the filtration surgery, and secondly, we used a 27 -gauge blunt needle to lift the scleral flap and viscoelastic materials to separate the sclera and conjunctiva. Because this needle revision via the anterior chamber involves blunt separation performed using a blunt needle and viscoelastic materials, intraoperative and postoperative complications were rare and the surgery was considered safe.

In cases of needle revision including those in which antimetabolites such as MMC or 5-FU are applied, many studies have reported surgical success rates of about $50 \%$ to $70 \%{ }^{5,6,10-12}$ The postoperative prognosis of needle revision in our institution was similar to those reported previously, despite many cases of severe secondary glaucoma treated without antimetabolites. In addition, few complications developed intraoperatively and postoperatively as our procedure was performed using a blunt needle and viscoelastic materials.

\section{Conclusion}

In conclusion, our new method of blunt needle revision is a safe and effective procedure that may be used to repeatedly treat cases of early failed filtering blebs after trabeculectomy, despite digital ocular massage and laser suture lysis, prior to performing transconjunctival needle revision using a sharp needle, particularly in the severe case such as rubeotic glaucoma. However, as this procedure was performed only in patients with early-stage disease and needle revision was done within the first month after the filtration surgery, the surgical outcomes of cases with postoperative terms exceeding 1 month should be evaluated. For cases in which this procedure is ineffective, conventional transconjunctival needle revision using a sharp needle may be a better choice for surgical intervention. 


\section{Disclosure}

All authors have no financial interest in any aspect of this work.

\section{References}

1. Costa VP, Correa MM, Kara-Jose N. Needling versus medical treatment in encapsulated blebs. A randomized, prospective study. Ophthalmology. 1997;104(8):1215-1220.

2. Azuara-Blanco A, Katz LJ. Dysfunctional filtering blebs. Surv Ophthalmol. 1998;43(2):93-126.

3. Singh J, Bell RW, Adams A, O'Brien C. Enhancement of post trabeculectomy bleb formation by laser suture lysis. $\mathrm{Br} J$ Ophthalmol. 1996;80(7):624-627.

4. Greenfield DS, Miller MP, Suner IJ, Palmberg PF. Needle elevation of the scleral flap for failing filtration blebs after trabeculectomy with mitomycin C. Am J Ophthalmol. 1996;122(2):195-204.

5. Rotchford AP, King AJ. Needling revision of trabeculectomies bleb morphology and long-term survival. Ophthalmology. 2008;115(7): 1148e4-1153e4.
6. Anand N, Khan A. Long-term outcomes of needle revision of trabeculectomy blebs with mitomycin $\mathrm{C}$ and 5-fluorouracil: a comparative safety and efficacy report. J Glaucoma. 2009;18(7):513-520.

7. Mardelli PG, Lederer CM Jr, Murray PL, Pastor SA, Hassanein KM. Slit-lamp needle revision of failed filtering blebs using mitomycin C. Ophthalmology. 1996;103(11):1946-1955.

8. Yokoyama M, Ishida K, Nakamura H. Revision of filtration from anterior chamber after trabeculectomy. Journal of the Eye. 2000; 17(10):1413-1416.

9. Pasternack JJ, Wand M, Shields MB, Abraham D. Needle revision of failed filtering blebs using 5-Fluorouracil and a combined ab-externo and ab-interno approach. $J$ Glaucoma. 2005;14(1):47-51.

10. Palejwala N, Ichhpujani P, Fakhraie G, Myers JS, Moster MR, Katz LJ. Single needle revision of failing filtration blebs: a retrospective comparative case series with 5-fluorouracil and mitomycin C. Eur J Ophthalmol. 2010;20(6):1026-1034.

11. Kapasi MS, Birt CM. The efficacy of 5-fluorouracil bleb needling performed 1 year or more posttrabeculectomy: a retrospective study. J Glaucoma. 2009;18(2):144-148.

12. Jacobs S, Gillis A, Van Malderen L, Zeyen T. Needling-revision of failed filtering blebs. Bull Soc Belge Ophtalmol. 2005;(297):59-64.
Clinical Ophthalmology

\section{Publish your work in this journal}

Clinical Ophthalmology is an international, peer-reviewed journal covering all subspecialties within ophthalmology. Key topics include: Optometry; Visual science; Pharmacology and drug therapy in eye diseases; Basic Sciences; Primary and Secondary eye care; Patient Safety and Quality of Care Improvements. This journal is indexed on

Submit your manuscript here: http://www.dovepress.com/clinical-ophthalmology-journal

\section{Dovepress}

PubMed Central and CAS, and is the official journal of The Society of Clinical Ophthalmology (SCO). The manuscript management system is completely online and includes a very quick and fair peer-review system, which is all easy to use. Visit http://www.dovepress.com/ testimonials.php to read real quotes from published authors. 